\title{
THE RATIONALITY OF CERTAIN CONTINUOUS CURVES
}

\author{
BY G. T. WHYBURN
}

1. Introduction. In this paper we establish the following theorem.

THEOREM. Every plane continuous curve $M$ every subcontinuum of which is a continuous curve is a rational curve. $\dagger$

We first state two lemmas, both of which are very easy to prove.

LEMMA 1. In order that any continuous curve $M$ should be a rational curve it is necessary and sufficient that every maximal cyclic curve of $M$ be a rational curve.

Lemma 2. If $P$ is any point of a plane continuous curve $M$, every subcontinuum of which is a continuous curve, then there exists a continuous curve $M^{*}$, also every subcontinuum of which is a continuous curve, which contains $M$ and such that $P$ is on the boundary of no complementary domain of $M^{*}$.

The necessity of the condition in Lemma 1 is obvious. The sufficiency is established by the following steps: (a) a continuum $M$ is a rational curve if and only if every two points $A$ and $B$ of $M$ can be separated in $M$ by a countable subset of $M$. (Menger, loc. cit., proves this where $A$ and $B$ are closed sets; a simple application of the Lindelöf Theorem suffices to prove the part not included in Menger's theorem); (b) if two points $A$ and $B$ of a continuous curve $M$ lying together in a maximal cyclic curve $C$ of $M$ are separated in $C$ by a closed subset $K$ of $C$, then $K$ also separates $A$ and $B$ in $M$, (because if not, then $A$ and $B$ lie together in a component $H$ of $M-K$, and since $H \cdot C$

$\dagger$ A continuum $M$ is said to be a rational curve if each point of $M$ is contained in arbitrarily small neighborhoods in $M$ with countable boundaries (see K. Menger, Grundzïge einer Theorie der Kurven, Mathematische Annalen, vol. 95 (1925), p. 277); or, in other words, for each point $P$ of $M$ and each $\epsilon>0, P$ can be $\epsilon$-separated in $M$ by a countable subset of $M$ (see P. Urysohn, Mémoire sur les multiplicités Cantoriennes, II Verhandelingen der Akademie Amsterdam, vol. 13, No. 4). 
is connected and contains $A+B$, this contradicts the assumption that $K$ separates $A$ and $B$ in $C$ ); (c) if two points $A$ and $B$ of a continuous curve $M$ do not lie together in a maximal cyclic curve of $M$, there exists at least one point of $M$ which separates $A$ and $B$ in $M$. Under our hypothesis it follows by (b) and (c) that every two points of $M$ can be separated in $M$ by a countable subset of $M$; and hence, by (a), $M$ is a rational curve. Lemma 2 is readily established by constructing, in each complementary domain $D$ of $M$ having the given point $P$ on its boundary, a sequence of arc-crosscuts converging to $P$ such that $P$ is a limit point of no single component of $D$ minus the sum of these crosscuts, and these are added to $M$ to form $M^{*}$.

2. Proof of the Theorem. Let $P$ be any point of $M$ and $\epsilon$ any positive number. By Lemma 1 we may assume $M$ to be cyclicly connected. And by Lemma 2, we may assume, also without loss of generality, that $P$ is on the boundary of no complementary domain of $M$; for if this is not the case, we consider the curve $M^{*}$ and show that $M^{*}$ is rational at $P$; and then since $M^{*} \supset M$, it follows that $M$ also is rational at $P$. By a theorem of the author's $\dagger$ there exists a simple closed curve $J$ of diameter $<\epsilon / 4$ enclosing $P$ and such that if $K$ denotes the sum of the boundaries of all those complementary domains of $M$ each of which has a boundary point on $J$, then $J \subset K$ and $\delta(K)<\epsilon / 4$. Since $K$ is a subcontinuum of $M$, it must be a continuous curve. Let $C$ be the maximal cyclic curve of $K$ containing $J$, and let $B$ be the boundary of the complementary domain of $C$ which contains $P$. Let $\left[I_{i}\right], i=1,2,3, \cdots$ be the collection of complementary domains of $M$ such that if, for each $i, J_{i}$ denotes the boundary of $I_{i}$ then $J_{i}$ belongs to $K$ and contains at least one point of $B$. Then since $B \subset K$ and every point of $K$ is a boundary point of at least one complementary domain of $M$, therefore $B \subset \sum_{1}^{\infty} J_{i}$. Since $C$ and $M$ are cyclicly connected, therefore each of the curves $B, J_{1}, J_{2}, J_{3}, \cdots$ is a simple closed curve. Now since $B \subset M$, it follows that, for each $i$, the interior $I_{i}$ of $J_{i}$

† Concerning the complementary domains of continua, Annals of Mathematics, vol. 29 (1928), pp. 399-411, Theorem 18.

$\ddagger$ G. T. Whyburn, Cyclicly connected continuous curves, Proceedings of the National Academy of Sciences, vol. 13 (1927), pp. 31-38, Theorem 10. 
lies either wholly within or wholly without $B$; and if, for any $i$, $I_{i}$ lies within $B$, then $B \cdot J_{i}$ consists of exactly one point. Thus if we divide the curves of the collection $\left[J_{i}\right]$ into two classes $G_{1}$ and $G_{2}$ such that, for each $i, J_{i}$ belongs to $G_{1}$ or to $G_{2}$ according as $B \cdot J_{i}$ consists of a single point or of more than one point, then the interior of every curve of $G_{2}$ lies wholly without $B$.

Now let $Q$ be a point at a distance $\geqq \epsilon$ from $P$. Then $Q$ is without every one of the curves $B, J_{1}, J_{2}, \cdots$. Let $J_{n_{1}}, J_{n_{2}}, \cdots$ be those curves of class $G_{2}$ each of which contains a point $A_{n_{i}}$ which is accessible from $Q$ by an arc having only $A_{n_{i}}$ in common with $\sum J_{i}$. For each $i$, let $X_{i}$ and $Y_{i}$ be the boundary points of the component of $J_{n_{i}}-J_{n_{i}} \cdot B$ which contains $A_{n_{i}}$. For each $i$, $I_{n i}+X_{i}+Y_{i}$ contains an arc $T_{i}$ from $X_{i}$ to $Y_{i}$. Let $H$ denote the closed point set $\overline{\sum T}_{i}$. Then clearly $H \subset \sum T_{i}+B$. Now if $E$ is any point of $H$ not in $\sum T_{i}$, it is easily seen that $E$ must be accessible from $Q$ by an arc having only $E$ in common with $\sum J_{i}$. And since $E$ belongs to no arc $T_{i}$, clearly it can then belong to no curve of class $G_{2}$. Hence it must belong to a curve of class $G_{1}$; and thus there exists an integer $j$ such that $E=B \cdot J_{j}$. Therefore the set of all such points $[E]$ is countable; and since, for each $i, T_{i} \cdot M=X_{i}+Y_{i}$, it follows that $H \cdot M$ is countable.

Now let $Z$ be any point at a distance $\geqq \epsilon$ from $P$. Then $Z$ and $Q$ lie in the same complementary domain of $H$, since $H$ lies in a circle with center $P$ and radius $\epsilon / 2$. However, the set $H$ must separate $P$ and $Z$. For if not, there exists an arc $Z P$ from $Z$ to $P$ with $H \cdot Z P=Z$. Let $V$ be the first point on $Z P$, in the order from $Z$ to $P$, which belongs to $B$. Since $Z V-V$ contains no point of any of the $\operatorname{arcs} T_{i}$, it follows that $V$ is accessible from $Q$ and belongs to no curve of class $G_{2}$. But since the set of all such points of $B$ is countable, it is readily seen that every such point is a limit point of $\sum T_{i}$ and hence belongs to $H$, contrary to supposition. Thus $H \epsilon$-separates $P$ in the plane, and since $H \cdot M$ is countable it follows that $M$ is rational at $P$, and thus our theorem is established.

In conclusion I will remark that the above proof depends very sharply upon properties of the plane, and I do not know whether or not the theorem is true in a space of more dimensions, but would be very much interested in a solution for such a space.

John Simon Guggenheim Memorial Foundation 